\title{
Design and implementation home security system and monitoring by using wireless sensor networks WSN/internet of things IoT
}

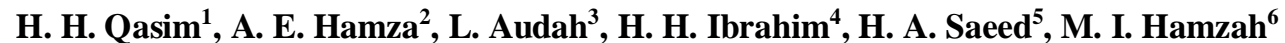 \\ 1,2,3,4,5 Wireless and Radio Science Centre (WARAS), Faculty of Electrical and Electronic Engineering, \\ Universiti Tun Hussein Onn Malaysia, Malaysia \\ ${ }^{2,4}$ Department of Engineering and Built Environment, Universiti Kebangsaan Malaysia, Malaysia \\ ${ }^{1,3}$ Department of Communications Engineering, Iraq University College, Iraq \\ ${ }^{6}$ Department of Computer Engineering University of Basrah, Iraq
}

\begin{tabular}{l} 
Article Info \\
\hline Article history: \\
Received Sep 6, 2019 \\
Revised Nov 4, 2019 \\
Accepted Nov 26, 2019 \\
\hline Keywords: \\
Arduino micro controller \\
Internet of things \\
Light dependant resistor \\
Smart car parking \\
Wireless sensor network
\end{tabular}

Article Info

Article history:

Received Sep 6, 2019

Revised Nov 4, 2019

Accepted Nov 26, 2019

Keywords:

Internet of things

Smart car parking

Wireless sensor network

\begin{abstract}
The dramatic advancments on communication and networking technologies have led to the emergence of Internet-of-Things (IoT). IoT technology has opened the door for various applications. In particular, the home automation was one of the common applications that took the advantage of IoT. Several research efforts have addressed the home automation system using IoT covering wide range of functionalities. One of the concerning tasks is providing a secure system that can give alarms for suspicious activities within the house. This paper presents a secure house system based on IoT where several activities are being sensed and detected. Specifically, gas, humidity, body temperature and motion have been considered within the sensing based on two main types of micro-controller including Arduino and Raspberry Pi. Consequentially, an Android prototype has bene developed in order to give an interactive interface for warning the house owner regarding any suscpicious activities. Results of simulation demonstrated the efficancy of the proposed system.
\end{abstract}

Copyright $(0) 2020$ Institute of Advanced Engineering and Science. All rights reserved.

\section{Corresponding Author:}

\section{H. H. Qasim,}

Wireless and Radio Science Centre (WARAS), Faculty of Electrical and Electronic Engineering,

Universiti Tun Hussein Onn Malaysia,

Batu Pahat, Johor, Malaysia.

Email: enghamza.iQ@gmail.com

\section{INTRODUCTION}

In the last decade, the advancements in communication and networking technologies are mainly concentrated on the Internet of Things (IoT) [1, 2]. In a real-world scenario, IoT-based services enhance the domestic environment and are exploited in numerous applications. Home automation based IoT is an imperative and common applications. In home automation, all home appliances are networked together which provides the capabilities to operate without human intervention. Home automation gives a significant change in people life which gives smart operating of home appliances [3, 4].

Numerous research efforts have been presented for proposing smart house system for example, Deng et al. [5] proposed a scheduling model for smart house based on optimization algorithm. The proposed algorithm has been intended to search for find the best solution of balancing the electricity consumption in order to reduce expenses. This has been represented by utilizing the Genetic Algorithm (GA) in order to find suitable load of consumption. Similarly, Singh et al. [6] proposed an efficient house management system based on micro-controller Ardunio sensors. The proposed systme aimed at sensing wide range of things including gas cylinder using LM35 sensors and IR sensors for electronic devices. The main goal is to reduce the energy consumption by sensing the presence of house owner. 
On the other hand, Rodrigues et al. [7] proposed a smart house system that is intended to reduce water consumption. The proposed system utilized severl sensors to observe the temperature, usage and availability of water inside the house. Using specific parameters and threshold, the proposed system will manage the consumption in water. For the accident prevention, Teslyuk [8] presented a smart house system that was intended to sense and observe events inside the house in order to prevent any kind of risk. The proposed system utilized various types of sensor where fire, gas or smoking and water leaking are being sensed using Arduino micro-controllers. Consequentially, the sensed information is being passsed into a neural network architecture that aimed at predicting the potential risk. Once the risk is being detected, an early alarm is being initiated.

Apart from sensing smoke, gazes or fires, Nath et al. [9] presented a novel method for smart house system where the sensing was based on voice-location detection. The proposed method has utilized Amazon Echo as a voice interface along with HC-SR04 ultrasonic sensor in order to detect the voice based location inside the house. Mahmoud et al. [10] presented a cloud-based approach for house automation. The proposed approach is based on a web portal that is being controlled by an ESP32 Wi-Fi module.

As noticed from the literature, the smart house system contains various aspects in which the room for improvement is open. One of the concerning issues is the security where there are wide range of activities that can be sensed. In addition, the type of sensors used in the literature for smart houses was energyconsuming. In order to prolong the management of any smart house system, an efficient sort of sensors must be examined. Therefore, this study aims to present a secure and smart house system based on Arduino and Raspberry Pi microcontroller in which humidity, gas, body temperature and moving objects are being detected. Finally, a prototype based on Android is being provided in order to collect the sensed information from the sensors for sending it to the house owner.

\section{RESEARCH METHOD}

There are multi-functions in this home security system .Wireless Sensors system consists of a set of sensors such as temperature, humidity, gas sensor (MQ-135), Flam sensor, two Arduino Uno and two Nrf24101 transceiver that is transmitter and receiver, If any sensor is sense from transmitter will be send signal wirelessly to receiver though Arduino and then send signal to raspberry pi to display the data on serial monitor through program of Arduino by idea Internet of thing. Another function for home is Motion detection with tracking camera system, in this system using two PIR sensors (motion sensor), Servo and Camera. When the thief will come inside the home the motion sensor will be detect and the camera automatically start taking image and upload the data on internet of thing through Raspberry pi. RFID system, in this system using Buzzer, RFID, Arduino Uno, two led green and red, The identification system will give the green light when the access card correct and allow to enter to the room .while cannot allow to access inside the room when the access card incorrect and give the red light with a warning voice and then the Arduino send signal to photon and then send SMS to the user via Internet of thing. The last one Key lock system in this system Consists keypad, servo, buzzer, relay and Arduino Uno .A permit to enter the house requires the entry of the passwordif the password is correct the door open, if enter password incorrect during three time, the system will send a signal photon via Arduino and then send SMS to the user of the house via the Internet. The block diagram of home security system is show below in Figure 1. The hardware of the system mainly comprises sensor section Arduino uno, raspberry pi, photon particle, RFID, Keypad, ip camera, LCD, Servo motor, and NRF24L01. Following sub-sections will tackle each sensor individually.

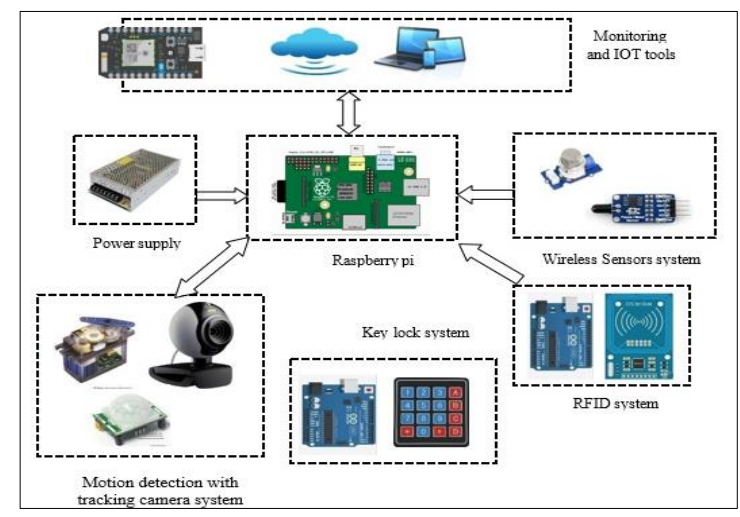

Figure 1. Block diagram of the proposed home security system 


\subsection{Arduino processing unit}

An Arduino board generally comprises of an Atmel 8-bit, 16-bit, or 32-bit AVR microcontroller (in spite of other vendors' microcontrollers have been used since 2015) with reciprocal components that can be easily programmed and incorporated into different circuits. Figure 2 illustrates the Arduino microcontroller. An essential part of the Arduino is its common connector, which allows the client to make interfacing between the CPU boards to various interchangeable extra modules (called shields). Most boards contain $5 \mathrm{~V}$ direct regulators and $16 \mathrm{MHz}$ crystal oscillators (or some ceramic resonators), although some boards (such as LilyPad) operate at $8 \mathrm{MHz}$ and due to specific shape factor restrictions, on-board voltage regulators are eliminated. Arduino's microcontrollers are additionally pre-programmed with boot loaders to simplify the uploading of desired programs to on-chip flash memory compared to different gadgets that regularly require a software engineer for programming purpose [11].

\subsection{Raspberry pi 3 mode $B$}

Raspberry Pi is a small single board PC propeller created by the Foundation of Raspberry Pi in the UK to promote schools and create basic programming engineering guidelines for the nations. As the Foundation of Raspberry Pi pointed out, greater than 5 million Raspberry Pis have been sold before Feb 2015, recording it the highest product in United Kingdom computers. At the end of November 2016, they had sold 11 million Raspberry Pi, and in March 2017 they had reached 12.5 million units, recording it the third "generally useful personal computer" ever. In July 2017, it announced that the total transaction volume has currently reached nearly 15 million units of Raspberry Pi [12]. Model B of raspberry is show in Figure 3.

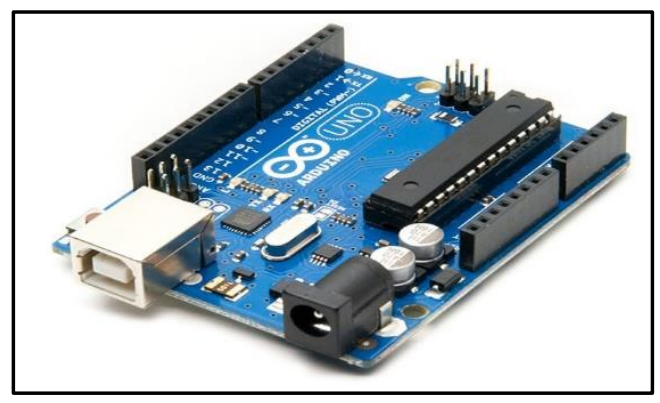

Figure 2. Arduino UNO microcontroller

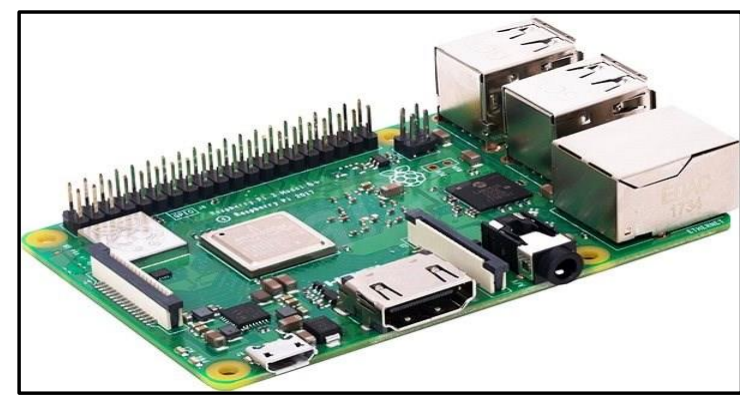

Figure 3. Model B of raspberry

\subsection{Spark photon}

Spark Photon microcontroller for IoT applications. The Photon has two buttons. The Reset button resets the Photon, restarting the processor. The Setup button ("Mode" button on the Core) is used in conjunction with the Reset button to allow more severe resetting of the Photon, including a full factory reset. The pins labeled D0 to D7 are general purpose GPIO pins that can act as either digital inputs or outputs. Some of these pins can also act as analog outputs (pins D0 to D4) using a technique called PWM (Pulse Width Modulation.) There is a blue LED next to pin D7 that is connected to D7. The Photon has a built-in chip antenna that will work fine in most WiFi situations, but the Photon also has a tiny antenna socket to which an external antenna can be attached. This is useful for extending the WiFi range of the device by adding a more sensitive or directional antenna [13]. The spark photon is show below in Figure 4.

\subsection{RFID system}

Radio Frequency Identification (RFID) innovation or essentially "tags" are little transponders that respond to queries from peruse by remotely transmitting serial numbers or comparable identifiers wirelessly $[14,15]$. It is relied upon that RFID tags will spread to billions of dollars throughout the following couple of years. However, they are handled in the same way as bar codes, regardless of the privacy implications of such advanced innovation. RF module IC card MFRC-522 RFID sensor module given in Figure 5. The primary benefits provided by RFID are quality clients' care, resource optimization, proficient business forms, greater precision, and powerful business and human services processes. RFID can help identify background knowledge and help to enhance the objects consistency of specific procedures. Nonetheless, it is important to study the use of RFID segments in a medical condition utilizing. The major components of RFID are readers, antennas and tags. The examination of these parts gives a comprehension of their utilization in the healthcare environment and their integration in the medical procedures. 


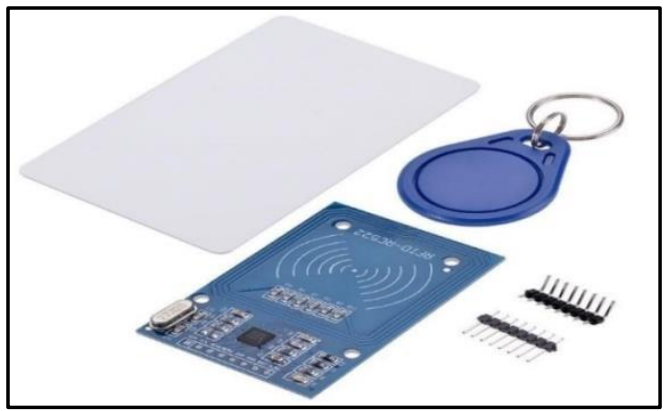

Figure 4. The spark photon

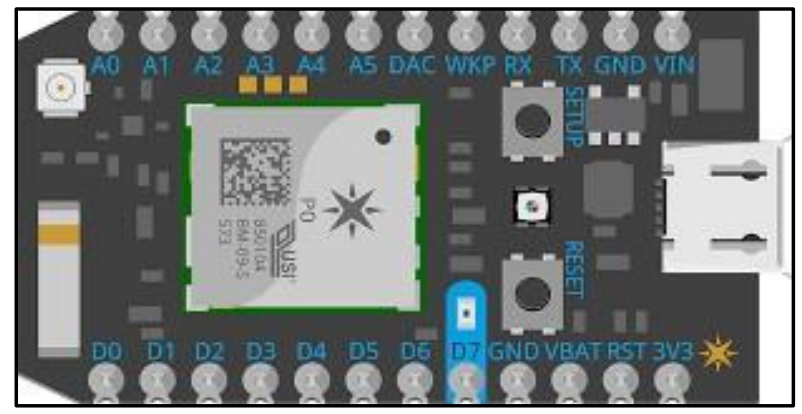

Figure 5. RFID sensing module

\subsection{PIR sensor}

The PIR sensor (passive infrared sensor) is an electronic sensor that measures infrared (IR) light emitted by objects in its field of view. They are frequently utilized as a part PIR-based movement identifiers [10]. At the point for an object for example a human goes throughout a background (for instance, a wall), the temperature in the sensor's field of view at this point will increase from normal temperature to body temperature and after that back to its original state. The sensor transfers over the subsequent change in the approaching infrared radiation into an adjustment in the yield voltage, and this triggers the recognition [16]. PIR has numerous configurations in various applications of uses. The most widely recognized models have multiple mirrored lenses or Fresnel lenses, with a powerful scope of approximately 10 meters (30 feet) and a field of view of fewer than 180 degrees. Figure 6 shows PIR sensor.

\subsection{DHT 11 sensor}

The digital temperature and humidity sensor (DHT11) is a composite Sensor includes an adjusted digital signal output of the dampness and temperature. Figures 3-9 demonstrate DHT11 sensor. Humidity sensors identify the related humidity of the specific conditions in where they are set. They measure all the moistness and temperature noticeable all around and show related humidity as a level of the proportion of the humidity noticeable all around to the most extreme sum which could be held in the surrounding atmosphere at the present temperature. As the air's temperature increase, it holds more dampness, therefore, the related humidity varies with the temperature [17].

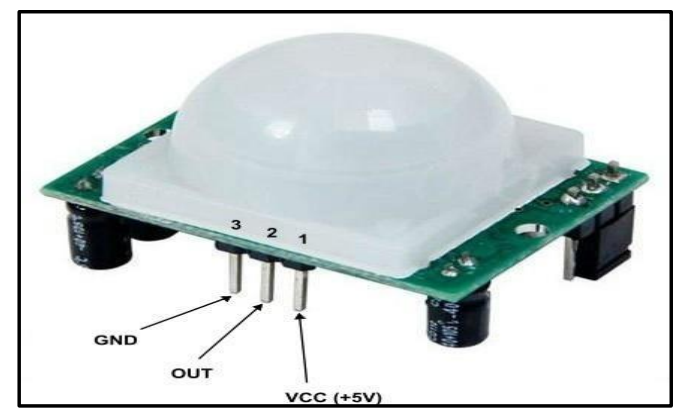

Figure 1. PIR sensor

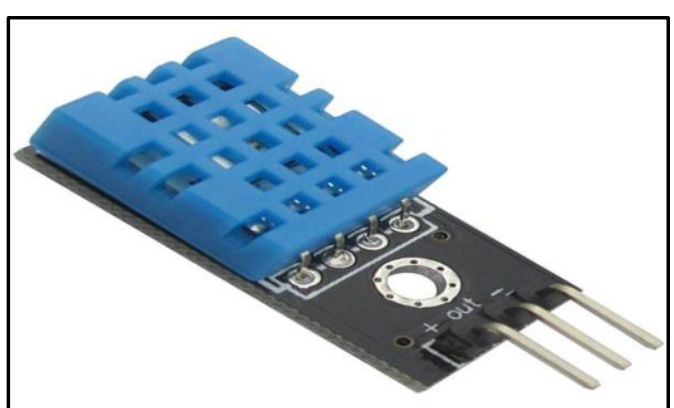

Figure 2. DHT 11sensor

\subsection{MQ-135 Gas sensor}

The (MQ-135) Gas Sensor module can be used to detect gas leaks (in houses and factories). It is appropriate for the recognizing of LPG, H2, CO, CH4, alcohol, Smoke or propane [18-20]. Because of its high affectability and quick reaction time, measurements can be made as fast as possible. The sensor's sensitivity can be balanced by utilizing a potentiometer. Gas leakage discovery is the way toward recognizing possibly unsafe gas leakage from the use of sensor. These detectors often use sound alerts to warn human when they detect unsafe gases. MQ-135sensor shows in Figure 8. 


\subsection{Flame sensor}

The flame sensor is a type of detectors that intended to detect and react to the presence of a fire or flame, permitting fire recognition [21, 22]. The response to the identified flame rely upon the establishment, yet may incorporate issuing an alert, deactivating the fuel line (for example, a petroleum gas line or propane) and starting the fire extinguishing system. Due to the usage of flame detection mechanism, flame sensors are usually faster and more Precise in respond than smoke or thermal sensors. Flame sensor shows in Figure 9.

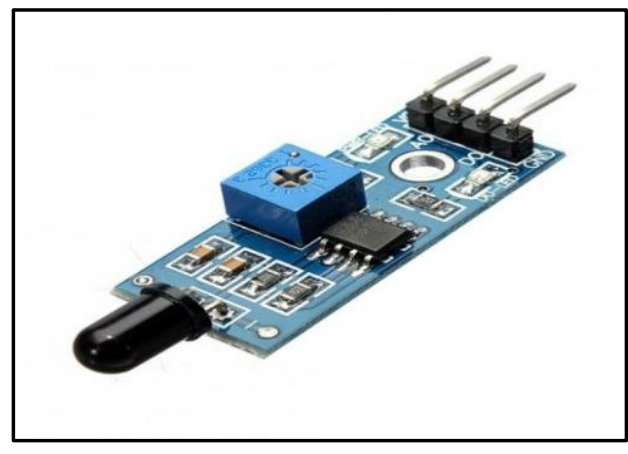

Figure 3. MQ-135sensor

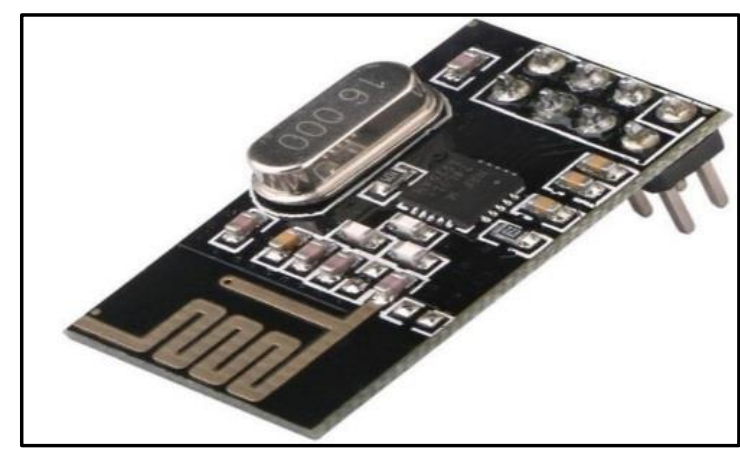

Figure 4. Flame sensor

\subsection{NRF24L01 transceiver}

An RF24L01 is a solitary -chip of $2.4 \mathrm{GHz}$ transceiver with a settled base and protocol motor intended for ultra-low energy wireless applications [23-25]. The nRF24L01 is intended for task of the worldwide ISM band of 2.400 to $2.4835 \mathrm{GHz}$. Designing a radio system using the nRF24L01 requires an MCU (microcontroller) and not very many outer passive parts. The nRF24L01 is designed and worked through the Serial Peripheral Interface (SPI). Through this interface, register maps can be used. GFSK modulation is utilized by the radio front end. It has client configurable parameters such as yield power, air information rate, and channel. The nRF24L01 supports an over-the-air information rate of $2 \mathrm{Mbps}$. Figure 10 shows NRF24L01 Transceiver.

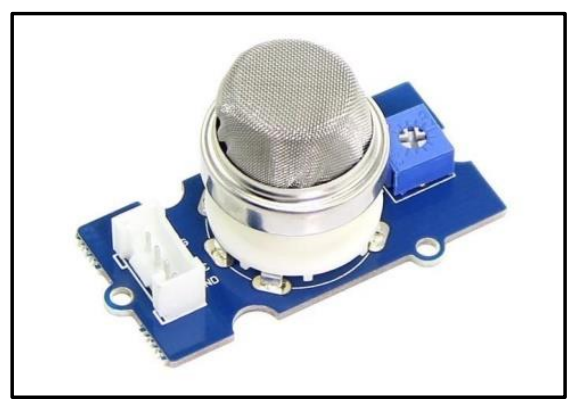

Figure 5. NRF24L01 transceiver

\section{RESULTS AND ANALYSIS}

\subsection{Implementation password lock system}

The door lock system based on password security consists of the Arduino, keybad, LCD and Servo motor to open and close the door depending on the numbers entered by the user as shown in Figure 11: The password number is $\left\{{ }^{\prime} \mathrm{A},{ }^{\prime}, \mathrm{B},{ }^{\prime}, \mathrm{C}^{\prime}, \mathrm{D}^{\prime},{ }^{\prime}, ', \mathrm{H}^{\prime}\right\}$ character. If the inter numbers are correct, the arduino will be instructed to open the door by using servo motor and if the inter numbers are incorrect for three attempts the arduino will not open the door and an immediate warning signal will be sent to the photon board for SMS alarm to the user through idea internet of thing (iot).

\subsection{Implementation RFID system}

Illustrates the identification system. Where it scans the cards and works to give the green light of the cards allowed as shown in Figure 12, while the cards that it did not recognize will running the red light 
with a warning voice as shown in Figure 13, then the RFID arduino sent alarm signal to the practical photon for SMS alarm based on IOT. Three cards has been considered for tasting this system, one has been considered for access denied while the other are for authorize access.

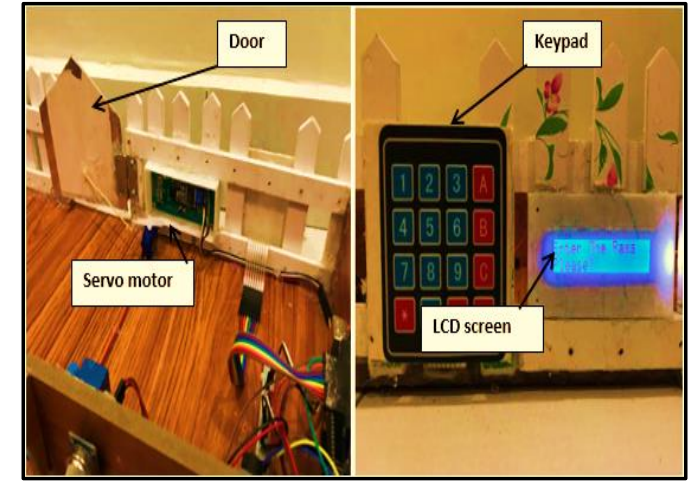

Figure 6. Implementation password lock system

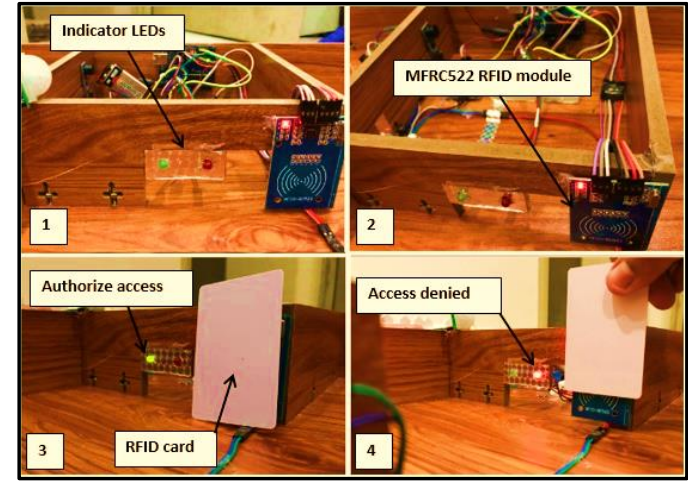

Figure 7. Implementation RFID system

\subsection{SMS alarm system using photon}

The photon is used to receive alarm alerts from RFID system and the door lock system where the photon will send it in the form of a text message to phone of the user based on the IoT and through IFTTT service based on web application. If this then that (IFTTT) is the free way to get all your apps and devices talking to each other. In addition to the web-based application, the service runs on iOS and Android SMS alarm system using photon from RFID system and The door lock system When we entered the wrong password for three consecutive times in the door lock system or tag in RFID by incorrect access card, the system send signal to particle photon to for processing and then the user received SMS text message through idea internet of thing (IOT). Show Figure 13 below the SMS that received in the phone.

\subsection{Implementation wireless sensors system}

The wireless sensors system consists from arduino uno, DHT11sensor, flame sensor, Gas sensor, nrf24101 transmitter and receiver, in Transmitter when I put gas lighter close to Gas sensor and also when I put fire close to flame sensor and if increase percentage of humidity and temperature of the air inside the room, In receiver the buzzer will be alarm when the arduino uno receive data of sensor from transmitter, the serial monitor window in arduino uno in IDE will show "gas alarm or flame alarm or percentage of humidity and temperature" if you remove gas lighter or fire and decrease the percentage of humidity and temperature in air that is mean the air is pure ,the buzzer will be turn off and serial monitor in window will be shown "no gas, no flame and percentage of humidity and temperature will be decrease" show below the Figure 14.

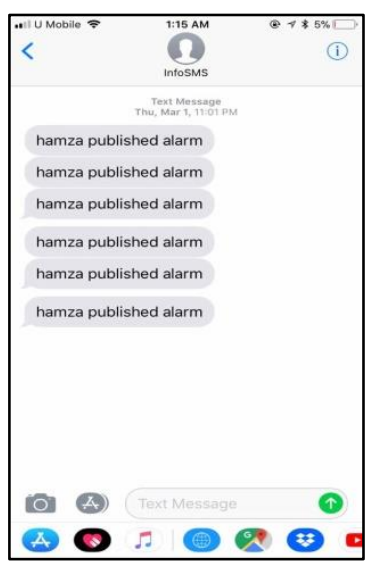

Figure 8. SMS alarm message

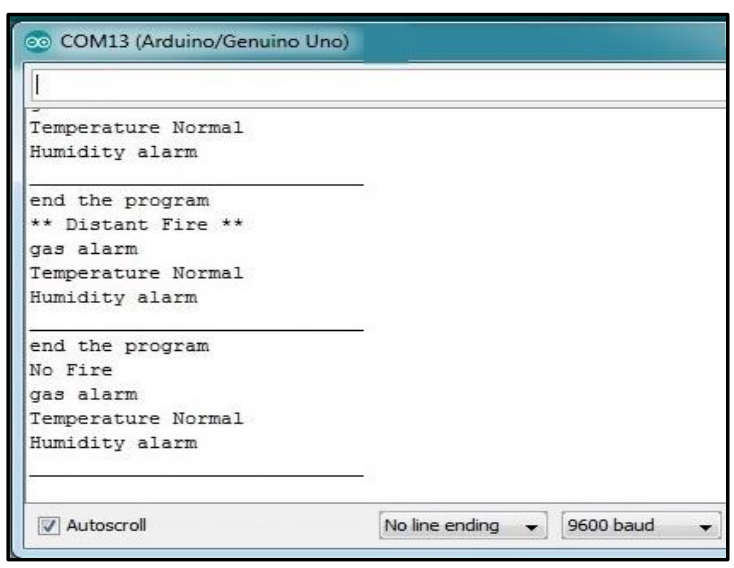

Figure 9. Wireless received data 


\subsection{Implementation motion detection system}

The motion detector system. This system consists of two PIRs sensor, one located in right side and the other on the left side. The servo motor moves the Camera to the right or left side depending on the motion detection in any direction. If the left side and the right side sensor detected to gather the servo move the camera left and right side and start automatically take image and save it on desktop of raspberry bi. The servo movement was adjusted from 45 to 135 degrees. In a situation where there is no movement, the position of the servo is in the middle at 90 decree. Raspberry PI camera record the detectable event based on two signals come from Arduino that moving the servo. A program has been written in python and saved in the desktop of the raspberry PI to run for recording at any time the user of the home wish to activate it. The user can monitor its home from any place using team viewer by any device such as phone or tablet. Implementation motion detection system shows in Figure 15.

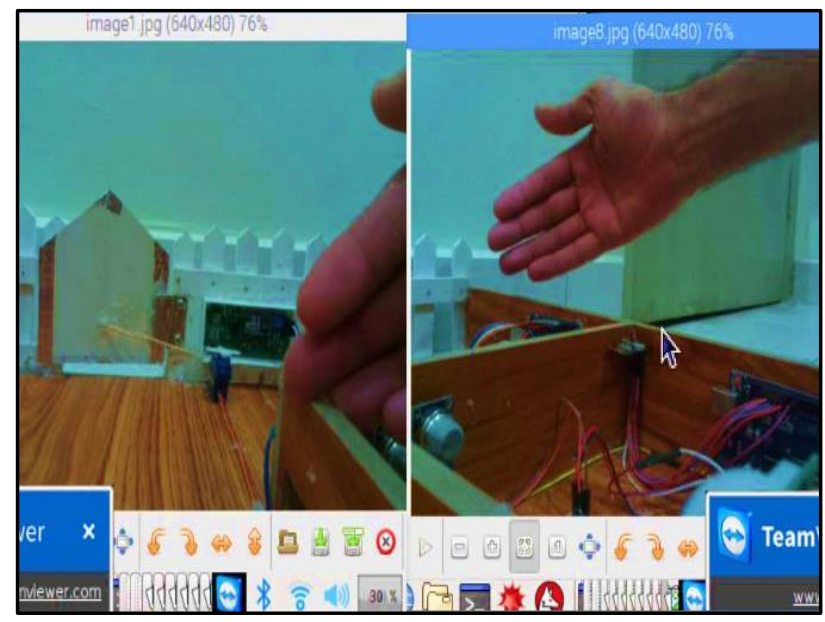

Figure 10. Motion detection

\section{CONCLUSION}

This paper has proposed a secured house model based on IoT. The proposed system aimed at sensing wide range of things including humidity, gas, body temperature and object motion. In addition, an interactive interface app has been developed in order to communicate, report and warn the house owner for any suspicious sensed activity. For future direction, extending the examination of activities that might occur within or around the house would be a potential research toward improving the home automation system.

\section{REFERENCES}

[1] A. Al-Fuqaha, M. Guizani, M. Mohammadi, M. Aledhari, and M. Ayyash, "Internet of things: A survey on enabling technologies, protocols, and applications," IEEE Communications Surveys \& Tutorials, vol. 17, no. 4, pp. 2347-2376, 2015.

[2] N. Rajput, "Internet of Things: Survey," Imperial Journal of Interdisciplinary Research, vol. 3, no. 6, 2017.

[3] D. Pavithra, and R. Balakrishnan, "IoT based monitoring and control system for home automation," 2015 Global Conference on Communication Technologies (GCCT), pp. 169-173, 2015.

[4] R. K. Kodali, V. Jain, S. Bose, and L. Boppana, "IoT based smart security and home automation system," 2016 International Conference on Computing, Communication and Automation (ICCCA), pp. 1286-1289, 2016.

[5] D. Changhong, Z. Sijie, Y. Wei, Y. Weiwei, T. Jin, and L. Zhengyi, "Two-Stage Optimization Model for Smart House Daily Scheduling Considering User Perceived Benefits," 2018 International Conference on Mathematics, Modelling, Simulation and Algorithms (MMSA 2018), pp. 64-68, 2018.

[6] H. Singh, V. Pallagani, V. Khandelwal, and U. Venkanna, "IoT based smart home automation system using sensor node," 2018 4th International Conference on Recent Advances in Information Technology (RAIT), pp. 1-5, 2018.

[7] R. R. Rodrigues, J. J. P. C. Rodrigues, M. A. A. d. Cruz, A. Khanna, and D. Gupta, "An IoT-based Automated Shower System for Smart Homes," 2018 International Conference on Advances in Computing, Communications and Informatics (ICACCI), pp. 254-258, 2018.

[8] V. Teslyuk, V. Beregovskyi, P. Denysyuk, T. Teslyuk, and A. Lozynskyi, "Development and implementation of the technical accident prevention subsystem for the smart home system," International Journal of Intelligent Systems and Applications, vol. 11, no. 1, pp. 1, 2018. 
[9] R. K. Nath, R. Bajpai, and H. Thapliyal, "IoT based indoor location detection system for smart home environment," 2018 IEEE International Conference on Consumer Electronics (ICCE), pp. 1-3, 2018.

[10] M. S. Mahamud, M. S. R. Zishan, S. I. Ahmad, A. R. Rahman, M. Hasan, and M. L. Rahman, "Domicile - An IoT Based Smart Home Automation System," 2019 International Conference on Robotics,Electrical and Signal Processing Techniques (ICREST), pp. 493-497, 2019.

[11] W. Dargie, and C. Poellabauer, "Fundamentals of wireless sensor networks: theory and practice," John Wiley \& Sons, 2010.

[12] Q. Jing, A. V. Vasilakos, J. Wan, J. Lu, and D. Qiu, "Security of the Internet of Things: perspectives and challenges," Wireless Networks, vol. 20, no. 8, pp. 2481-2501, 2014.

[13] S. Monk, "Getting Started with the Photon: Making Things with the Affordable, Compact, Hackable WiFi Module," Maker Media, Inc., 2015.

[14] S. Ravi, A. David, and M. Imaduddin, "Controlling \& Calibrating Vehicle-Related Issues Using RFID Technology," International Journal of Mechanical and Production Engineering Research and Development, vol. 8, no. 2, pp. 1125-1132, 2018.

[15] F. Xiao, Z. Wang, N. Ye, R. Wang, and X.-Y. Li, "One more tag enables fine-grained RFID localization and tracking," IEEE/ACM Transactions on Networking (TON), vol. 26, no. 1, pp. 161-174, 2018.

[16] M. Kaur, M. Sandhu, N. Mohan, and P. S. Sandhu, "RFID technology principles, advantages, limitations \& its applications," International Journal of Computer and Electrical Engineering, vol. 3, no. 1, pp. 151, 2011.

[17] P. Gupta, and J. Chhabra, "IoT based Smart Home design using power and security management," 2016 International Conference on Innovation and Challenges in Cyber Security (ICICCS-INBUSH), pp. 6-10, 2016.

[18] N. A. Sabuag, J. Ricohermoso, M. J. Amper, R. A. Espino, and R. Luna, "Ambient Air Quality Measurement using ATmega328 Microcontroller and MQ-135 Gas Sensor for Vehicular Emission Detection along commercial roads of Metro Manila," [2019-MADRID] Congreso Internacional de Tecnología, Ciencia y Sociedad, 2019"

[19] S. Kalpana, and A. L. Baghyam, "Electronic-Nose System for Classification of Fruits and Freshness Measurement using K-NN Algorithm," International Journal of Advanced Science and Technology, vol. 127, no. 1, pp. 88-91, 2019.

[20] X. Lu, J. Wang, G. Lu, B. Lin, M. Chang, W. He, "Quality level identification of West Lake Longjing green tea using electronic nose," Sensors and Actuators B: Chemical, vol. 301, pp. 127056, 2019.

[21] K. Grogan, Z. LaBry, P. N. Shah, and S. V. Sardeshmukh, "Dynamic Flame Thickening through In-Situ Numerical Stiffness Detection," AIAA SciTech Forum, pp. 1-11, 2019.

[22] A. Crompton, K. Gamage, S. Bell, A. Wilson, A. Jenkins, and D. Trivedi, "Gas Flow to Enhance the Detection of Alpha-Induced Air Radioluminescence Based on a UVTron Flame Sensor," Sensors, vol. 18, no. 6, pp. 1842, 2018.

[23] L. Cui, M. Qiu, H. Fan, L. Wang, S. Zhao, and J. Du, "Design and Research of Water Quality Monitoring System Based on nRF24L01 Wireless Communication," IOP Conference Series Earth and Environmental Science, vol. 208, no. 1, pp. 1-6, 2018.

[24] S. Aravinth Raj, and V. Venkatesh, "Implementation of Wireless Sensor Network with Low Cost and Low Power using Arduino and nRF24L01," International Journal of Pure and Applied Mathematics, vol. 119, no. 18, pp. 2095-2103, 2018.

[25] A. B. Bakri, R. Adnan, and F. A. Ruslan, "Wireless Hand Gesture Controlled Robotic Arm Via NRF24L01 Transceiver," 2019 IEEE 9th Symposium on Computer Applications \& Industrial Electronics (ISCAIE), pp. 16-22, 2019. 